\title{
Les nouveaux défis du théâtre du vingt-et-unième siècle
}

Patricia Corbier, MCF Langues et littérature anglaises et anglo-saxonnes

Le théâtre est un champ de forces très petit mais où se joue toujours l'histoire de la société, et qui, malgré son exiguïté, sert de modèle à la vie des gens, spectateurs ou pas. Laboratoire des conduites humaines, conservatoire des gestes et des voix, lieu d'expérience pour de nouveaux gestes, de nouvelles façons de dire.

George Banu

Propice à l'expérimentation, ce laboratoire incite les auteurs, acteurs, metteurs en scène et spectateurs à échanger leurs expériences et leurs représentations de la réalité. Dans la première moitié du vingtième siècle, les principes de représentation et d'interprétation, de drame et de réception ont été mis en question par les avant-gardes et tous les procédés esthétiques ont été testés jusqu'à leur extrême limite. A l'ère du vingt-et-unième siècle, une vision plus complexe du monde réclame des techniques innovantes et reconsidère la validation du vocable «dramaturgie » lui-même. Face à cette évolution permanente qui entraînera une redéfinition des stratégies et objectifs de l'art théâtral, je questionnerai le pôle de la représentation et les conditions de la réception.

Suite à la chute des idéologies, la société occidentale est entrée dans la postmodernité. Après le théâtre de l'Absurde et le théâtre existentialiste, la dénomination de " théâtre postmoderne »s'est appliquée au théâtre à partir de la fin des années cinquante avec l'émergence de la « performance » et du « happening ». Pour éviter tout malentendu, il serait utile de préciser que, pour le théoricien américain, le "postmoderne" naît au début des années soixante tandis que, pour le théoricien français, il apparaît au début des années quatre-vingt. « Comme 'moderne' ou 'contemporain' le terme 'postmoderne' traverse l'ensemble du champ des représentations pour qualifier notamment architecture, danse, théâtre, littérature, arts visuels. Comme ces termes, il est donc avant toute chose une catégorie esthétique marquant l'historicité d'une époque » (Ruffel 2). Il relève d'une attitude qui questionne le credo moderniste mais les différents contextes auxquels il a été assimilé et ses diverses ramifications ont donné lieu à d'innombrables réévaluations. Peu employé par la critique dramatique en raison de son manque de rigueur théorique, le terme sert à décrire un style de jeu, une attitude de production et de réception. L'un des apports du postmodernisme touche à la renégociation du rapport entre l'œuvre et le spectateur : «Les pôles de la réception et de la perception sont 
survalorisés. L'œuvre postmoderne n'a d'autre référence qu'à elle-même ; elle n'est plus qu'une dérive des signes » (Pavis 264). La terminologie théâtre " postdramatique » choisie par Hans-Thies Lehman apporte plus de précisions pour définir ce nouveau théâtre. Dans $L e$ théâtre postdramatique, Lehman présente des réflexions et analyses de manifestations théâtrales contemporaines diverses en Europe et en Amérique à partir des années 1970 qui se détournent du modèle dramatique traditionnel, dans une perspective à la fois esthétique et historique. Pour l'auteur, les termes utilisés pour définir ce nouveau théâtre - « célébration de l'art comme fiction, célébration du théâtre comme processus jugé autoritaire et/ou archaïque, discontinuité, hétérogénéité, non-textualité, pluralisme, plusieurs codes, subversion, multilocalisation, perversion... » (32) - s'avèrent peu pertinents pris individuellement et sujets à contradiction. L'épithète " postdramatique », quant à lui, s'applique à un théâtre amené à opérer au-delà du drame et « englobe l'actualité / la reprise / la continuité d'esthétiques anciennes, par exemple de celles qui, autrefois déjà, s'étaient distanciées de l'idée dramatique au niveau du texte et du théâtre. L'art ne peut en aucune manière se développer sans rapport à des formes antérieures. Le seul critère est le niveau, la lucidité et la forme particulière de ce rapport » (36). La visée de Lehman dans son ouvrage est d'aider à comprendre les conditions et implications tant esthétiques que théoriques du spectacle théâtral, plutôt que des textes depuis 1970. L'œuvre devient unique et le spectateur est confronté à un événement. L'auteur accorde également une grande attention à la perception des œuvres par les spectateurs. Son essai consiste à présenter « l'articulation d'une logique esthétique » du nouveau théâtre.

Imprégnée de cette mouvance, Erika Fisher-Lichte aborde plus récemment dans The Transformative Power of Performance. A New Aesthetics une nouvelle esthétique qui se concentre sur la performance comme expérience d'événements et sensations et sur les procédés utilisés plutôt que sur le produit. Elle rejoint ainsi la philosophie anglo-saxonne de la performance qui envisage le théâtre comme un événement vivant et qui s'intéresse à l'interaction entre comédiens et spectateurs.

Cette mutation aux aspects syncrétiques présente des formes singulières de spectacle qui remettent en question l'illusion théâtrale et les supports d'énonciation. Une indistinction s'organise entre le théâtre et la présence directe de la vie. Alain Badiou décrit ce phénomène comme « un théâtre sans théâtre... une présence du jeu au ras de ce qui n'est pas joué ou n'est que le jeu social ordinaire » (21). Comme cela était déjà le cas chez Antonin Artaud ou le Living Theatre, le théâtre tend à abolir la représentation pour « devenir une monstration » et toute performance artistique évite de verser dans la théâtralité. Les performeurs-euses refusent la technique, ils choisissent de ne pas jouer, ne pas prétendre être quelqu'un d'autre ils préfèrent 
travailler avec un minimum d'artifices et leurs émotions propres. Ce qui importe, c'est dans la recherche de l'ici et du maintenant, de saisir une qualité de présence. Cette recherche n'est pas sans rappeler le Living Theatre des années cinquante dont l'objectif était de substituer au verbe le jeu du corps et de ses mouvements et de faire partager au spectateur l'immédiateté de l'expérience proposée. Nous retrouvons également les caractéristiques de la forme dramatique du Happening des années soixante qui proposait un spectacle qui invitait à la réflexion sociopolitique, aux questions de société au sens large sans texte préétabli. Son initiateur Allan, Kaprow, préférait le terme « événement » à « spectacle »; ce qui importait c'est ce qui arrivait (« happen ») par hasard, sans volonté particulière. Le Happening reposait sur quatre principes de base : les événements se déroulaient simultanément comme dans la vie; le public comprenait tous les actants (spectateurs et intervenants); l'aire de jeu s'étendait parmi les spectateurs, sans séparation; et le jeu était à base d'improvisations. Nous pouvons nous questionner sur la résurgence de ces anciennes formes dans le théâtre du ving-et-unième siècle.

Le théâtre postdramatique souligne un rapport conflictuel entre «spectacle» et « performance ». Le choix du vocable «performance» au sens donné par Jacques Scherer privilégie la relation, l'action et l'interaction. La performance artistique propose une expérience unique de partage d'une action, d'un espace, d'un temps et, en général, elle n'a lieu qu'une fois pour un public donné. Elle comporte une dimension expérimentale et rejette les effets de la dramaturgie, notamment le texte et la mise en scène : « Au lieu de représenter une histoire avec des personnages qui apparaissent et disparaissent en fonction de la psycho-logique de la narration, ce théâtre est fragmentaire, combine des styles disparates et s'inscrit dans une dynamique de la transgression des genres. Des formes singulières de spectacle inclassables émergent » (Lehman 3). Ce théâtre postdramatique qui donne lieu à de nouvelles pratiques théâtrales s'est manifesté « dans le sillage du développement, puis de l'omniprésence des médias dans la vie quotidienne depuis les années $1970 »(28)$. Actuellement, tous les arts du visible et du son semblent confondus et toute représentation est critiquée. La pratique artistique se présente dans sa dimension la plus expérimentale, délaissant l'héritage historique et la projection dans l'avenir pour explorer le présent. L'espace de représentation vise à susciter un questionnement sur le spectacle plutôt que d'élaborer des stratégies de condamnation. La scène devient ainsi un laboratoire où s'échangent idées et questions liées à l'humanité d'aujourd'hui et les discours et actions sont partagés avec le public sans valeur moralisatrice. Le texte perd ainsi sa singularité au profit de montage de textes non théâtraux et d'une écriture de plateau. Cette écriture redéfinit le métier de « mise en scène » en abolissant la hiérarchie entre le texte et la scène. La tâche de « l'écrivain de plateau » consiste à réunir écritures textuelle et scénique 
dans un même geste jusqu'à la matérialisation du projet en public. Tandis que dans le théâtre classique le rapport au texte était primordial, dans le théâtre postdramatique le texte devient un élément constitutif d'un tout où se côtoient le gestuel, le musical et le visuel. La question du texte de théâtre a toujours donné lieu à maints débats et la « révolution moderniste » du théâtre a remis en cause le «textocentrisme ». A l'instar de Bernard Dort, suite au passage du « textocentrisme » au « scénocentrisme », une nouvelle " révolution einsteinienne » consiste en « une relativisation généralisée des facteurs de la représentation théâtrale, les uns par rapport aux autres »(270). Cette proposition qui vise à abandonner tout centrisme a le mérite d'envisager le théâtre comme travail collectif, comme un système composé d'une multitude d'éléments, de médias variés agissant les uns sur les autres à des degrés divers. Ainsi, l'écrit ne sera qu'un des éléments de l'agencement de l'événement théâtral global. Le rôle de l'acteur n'est plus de répéter les répliques de l'auteur : «La scène est considérée comme point de départ et non comme lieu de re-copiage » (Lehman 43). Le théâtre de «situations » et le théâtre «psychologique » tendent à disparaître. Se trouve de ce fait mise en question la validité du vocable «dramaturgie » qui sous-entend que la lecture littéraire domine le théâtre et qui s'applique à l'art de la composition. Généralement, elle prend en compte la forme, l'esthétique et les motifs inhérents au travail du texte par le dramaturge. Face à l'émergence du théâtre contemporain riche de pratiques diversifiées et innovantes ne serait-il pas plus adéquat de parler de « dramaturgie visuelle » qui « ne signifie pas ici une dramaturgie organisée exclusivement de manière visuelle, mais une conception qui ne se subordonne pas au texte et peut développer librement la logique qui est la sienne » (147). Ce qui importe c'est que les divers matériaux choisis pour l'élaboration de l'œuvre théâtrale puissent se doter d'une signification. Les nouvelles technologies jouent une large part dans cette naissance du théâtre qui pouvait paraitre obsolète après la domination de l'image.

Au cours de l'histoire, diverses sortes de médias ont joué le rôle d'innovateurs dans le monde artistique. Depuis les années quatre-vingt-dix, grâce à l'essor des nouvelles technologies, le théâtre a la possibilité d'avoir recours à l'image et au son en temps réel. Ces avancées émanent de techniciens qualifiés et d'un équipement informatisé. Il est essentiel aujourd'hui de pouvoir utiliser les médias qui imprègnent largement les comportements humains afin de rendre compte du «réel» de nos sociétés à travers des représentations objectives de ce qui existe. D'autre part, les potentialités d'invention d'espaces scéniques de ces nouvelles technologies sont inépuisables et pourront transformer la sensibilité, l'intelligence humaine et également l'ensemble des arts. Pouvons-nous cependant définir ce 
nouveau modèle de composition théâtrale par le terme «médiaturgie », le média impliquant l'idée d'un procédé de communication d'un message sonore ou audiovisuel ?

Avec l'émergence du numérique et la capacité de l'ordinateur à traiter le son et l'image, l'écriture peut intégrer des médias différents et c'est donc tout le processus de composition de l'œuvre théâtrale qui se trouve transformé. Le texte cependant ne perd pas sa valeur, il présente la garantie que le théâtre ne sera éclipsé ni par la danse ni par l'image. Des pratiques innovantes à mettre en œuvre se font jour, offrant des possibilités jusque-là insoupçonnées telles que la conception d'agencements de textes aux effets démultipliés. Tout en se donnant pour but de mettre en lumière ce qui relève du « réel », cette nouvelle composition propre à l'art théâtral associe la pensée critique de la dramaturgie aux techniques médiatiques de l'illusion : « Le spectacle est saisissement d'une image et d'un sens qui frappent au cœur de l'esprit et qui le captent par le corps et la voix » (Bonté 50). Ces nouveaux outils virtuels, tout en stimulant l'imaginaire du récepteur, vont lui permettre de mieux appréhender le monde qui l'entoure, de se débarrasser de ses illusions et de mieux saisir sa vérité humaine.

Le spectacle s'adresse à tous les sens et élargit nos plans d'observation grâce à la diversité de ses composantes. Le récepteur bénéficie ainsi d'une multiplicité de points de vue. L'immersion sensorielle est favorisée grâce à un environnement technologique qui sollicite la dimension perceptive du public, ses perceptions auditives et visuelles. Ainsi, le concept d'immersion cognitive et physique place l'expérience du spectateur au cœur de la création. La subjectivité du récepteur est donc l'enjeu essentiel et l'articulation identification / distance convoquée par tout spectacle théâtral se trouve au centre même du corps sensible du récepteur. Le récepteur est intégré physiquement dans un environnement équipé d'installations qui intensifient ses perceptions corporelles. La construction spectaculaire dramatique créatrice de l'illusion théâtrale se trouve donc modifiée et ne s'adresse plus uniquement au regard. Nous sommes en présence d'une réception active et non contrainte.

En fait, quels que soient les moyens utilisés et les buts qui peuvent être radicalement différents - engagement total du spectateur et participation ou prise de distance - la fonction du théâtre est de proposer une ouverture sur la réalité et un déconditionnement. En effet, la représentation théâtrale œuvre pour faciliter une mise en question de notre vision du monde et une rupture avec les représentations que nous nous faisons de notre société. Et cette collaboration entre la scène et la salle permet ainsi de partager une expérience relative à notre société. En ce sens elle rappelle la fonction politique, civique et morale du théâtre grec qui visait à édifier tout citoyen membre de la Polis. 
La représentation est le fruit d'une construction collective qui engage auteurs, acteurs, metteurs en scène, techniciens et spectateurs. Le sens va ainsi se constituer à travers tous les éléments du spectacle qui composent la dramaturgie. Le théâtre du vingt-et-unième siècle, tout en restant attaché à des valeurs essentielles, est confronté à de nouvelles modalités de production qui nécessitent une pluridisciplinarité : une association de savoirs artistiques et techniques, et des pratiques et expérimentations sur plateau. Ces nouveaux procédés représentent des outils qui permettent l'exploration de la réalité et facilitent la communication avec un public. Des performances expérimentales multimédia ont ainsi recours à des projections vidéo qui alternent avec des événements sur scène, des interactions entre les acteurs sur scène et les acteurs à l'écran, et des simulations sophistiquées de réalité virtuelle qui incluent une dimension interactive. Les technologies numériques et Internet ont ainsi bouleversé les traditions artistiques et exigent une expertise dans le domaine technique qui peut apparaître comme un frein à ce développement. Le recours à la réalité virtuelle pour communiquer et expérimenter des idées représente une des plus récentes innovations. Ce phénomène de réalité augmentée permet de partager une expérience émotionnelle et de créer une illusion immersive. Dans le même temps, en raison de l'évolution de la société, les attentes du public se sont modifiées, créant ainsi de nouveaux défis. En effet, il s'agit moins pour le spectateur d'assister à un événement artistique que de partager une expérience positive de haute qualité en s'engageant totalement. Le public peut même interférer et collaborer à la production théâtrale finale selon son histoire et sa personnalité lui offrant ainsi une autre liberté, d'autres alternatives à son quotidien en dépassant le cadre de la représentation traditionnelle. La dramaticité se trouve, de ce fait, reconfigurée à travers les interactions entre les acteurs sur la scène et les images créées par ordinateur d'une part, ainsi qu'à travers les interactions entre l'action sur la scène et le public qui bénéficie d'une expérience participative, d'autre part.

Les modalités de création, de production et de distribution évoluent et se transforment en relation avec l'avènement d'une société numérique. La complexité inhérente à ces innovations, caractérisée par la professionnalisation et les contraintes d'ordre économique, représente certes de nouveaux enjeux et des conditions d'élaboration différentes, le texte ne représentant qu'une partie d'un ensemble qui doit être adapté à travers un travail collectif et collaboratif. Ces nouveaux outils offrent une autre façon d'interroger le monde, le réel et la réalité, la condition humaine que le théâtre n'a cessé, au cours de son histoire, de placer au centre de ses préoccupations. En effet, la diversité des nouvelles stratégies de l'art théâtral accompagne et continue d'enrichir nos perceptions intellectuelles et sensibles du monde qui nous entoure. 


\section{Ouvrages cités}

Badiou, Alain. Eloge du théâtre. Paris : Flammarion, 2013.

Bonté, Patrick. «La scène de la philosophie est une philosophie sans scène ». Alternatives théâtrales, Philoscène : la philosophie à l'épreuve du plateau 135 (2018).

Dort, Bernard. Théâtres. Paris : Seuil, 1986.

Fisher-Lichte, Erika. The Transformative Power of Performance. A New Aesthetics. Londres : Routledge, 2004.

Lehman, Hans-Thies. Le théâtre postdramatique. Paris : L'Arche, 2002.

Pavis, Patrice. Vers une théorie de la pratique théâtrale. Voix et images de la scène. Lille : PU Septentrion, 2000.

Ruffel, Lionel. «Postmoderne ». Le lexique socius. Dir. Anthony Glinoer et Denis SaintAmand. http://ressources-socius.info/index.php/lexique/21-lexique/61-postmoderne 